\title{
1 Retinal Age as a Predictive Biomarker for Mortality Risk
}

2

3 Running title: Retinal age predicts mortality

4

5 Authors

6 Zhuoting Zhu, MD PhD ${ }^{1}$

7 Danli Shi, MD $^{2}$

8 Guankai Peng, $\mathrm{BS}^{3}$

9 Zachary Tan, MBBS, MMed, MMSc ${ }^{4}$

10 Xianwen Shang, $\mathrm{PhD}^{1}$

11 Wenyi Hu, MBBS ${ }^{1}$

12 Huan Liao, MD ${ }^{5}$

13 Xueli Zhang, $\mathrm{PhD}^{1}$

14 Yu Huang, MD $\mathrm{PhD}^{1}$

15 Honghua $\mathrm{Yu}, \mathrm{MD} \mathrm{PhD}^{1}$

16 Wei Meng, $\mathrm{BS}^{3}$

17 Wei Wang, MD $\mathrm{PhD}^{2}$

18 Xiaohong Yang, MD $\mathrm{PhD}^{1}$

19 Mingguang $\mathrm{He}, \mathrm{MD} \mathrm{PhD}^{1,2,4,6}$

20

\section{Affiliations}

22 1. Department of Ophthalmology, Guangdong Academy of Medical Sciences, 23 Guangdong Provincial People's Hospital, Guangzhou, China.

24 2. State Key Laboratory of Ophthalmology, Zhongshan Ophthalmic Center, Sun 25 Yat-sen University, Guangzhou, China.

26 3. Guangzhou Vision Tech Medical Technology Co., Ltd.

27 4. Centre for Eye Research Australia; Ophthalmology, University of Melbourne, 28 Melbourne, Australia.

29 5. Neural Regeneration Group, Institute of Reconstructive Neurobiology, University 30 of Bonn, Bonn, Germany. 
medRxiv preprint doi: https://doi.org/10.1101/2020.12.24.20248817; this version posted December 30, 2020. The copyright holder for this preprint (which was not certified by peer review) is the author/funder, who has granted medRxiv a license to display the preprint in perpetuity. It is made available under a CC-BY-NC-ND 4.0 International license .

31 6. Ophthalmology, Department of Surgery, University of Melbourne, Melbourne, $32 \quad$ Australia

33

34 Word count: Summary: 249; Research in context: 286; Whole paper: 2549.

35 Tables: 2; Figures: 5.

36

37 Corresponding author

38 Mingguang $\mathrm{He}, \mathrm{MD} \mathrm{PhD}$

39 Email: mingguang.he@unimelb.edu.au

40 Xiaohong Yang, MD PhD

41 Email: syyangxh@scut.edu.cn

42 Wei Wang, MD PhD

43 Email:zoc wangwei@yahoo.com 


\section{Summary}

45 Background

46 Ageing varies substantially, thus an accurate quantification of ageing is important. We

47 developed a deep learning (DL) model that predicted age from fundus images (retinal 48 age). We investigated the association between retinal age gap (retinal 49 age-chronological age) and mortality risk in a population-based sample of 50 middle-aged and elderly adults.

\section{Methods}

52 The DL model was trained, validated and tested on 46,834, 15,612 and 8,212 fundus

53 images respectively from participants of the UK Biobank study alive on $28^{\text {th }}$ February 54 2018. Retinal age gap was calculated for participants in the test $(n=8,212)$ and death $55(\mathrm{n}=1,117)$ datasets. Cox regression models were used to assess association between 56 retinal age gap and mortality risk. A restricted cubic spline analyses was conducted to 57 investigate possible non-linear association between retinal age gap and mortality risk.

\section{$58 \quad$ Findings}

59 The DL model achieved a strong correlation of $0.83(\mathrm{P}<0.001)$ between retinal age 60 and chronological age, and an overall mean absolute error of 3.50 years. Cox 61 regression models showed that each one-year increase in the retinal age gap was associated with a $2 \%$ increase in mortality risk (hazard ratio $=1 \cdot 02,95 \%$ confidence

63 interval:1·00-1·04, P=0.021). Restricted cubic spline analyses showed a non-linear 64 relationship between retinal age gap and mortality $\left(\mathrm{P}_{\text {non-linear }}=0 \cdot 001\right)$. Higher retinal 65 age gaps were associated with substantially increased risks of mortality, but only if the gap exceeded 3.71 years.

\section{Interpretation}

68 Our findings indicate that retinal age gap is a robust biomarker of ageing that is 69 closely related to risk of mortality.

\section{$70 \quad$ Funding}

71 National Health and Medical Research Council Investigator Grant, Science and 72 Technology Program of Guangzhou. 
medRxiv preprint doi: https://doi.org/10.1101/2020.12.24.20248817; this version posted December 30, 2020. The copyright holder for this preprint (which was not certified by peer review) is the author/funder, who has granted medRxiv a license to display the preprint in perpetuity.

It is made available under a CC-BY-NC-ND 4.0 International license .

74 Key words: retinal age, mortality, prediction 


\section{Research in context}

\section{Evidence before this study}

77 Ageing at an individual level is heterogeneous. An accurate quantification of the 78 biological ageing process is significant for risk stratification and delivery of tailored 79 interventions. To date, cell-, molecular-, and imaging-based biomarkers have been 80 developed, such as epigenetic clock, brain age and facial age. While the invasiveness 81 of cellular and molecular ageing biomarkers, high cost and time-consuming nature of neuroimaging and facial ages, as well as ethical and privacy concerns of facial

83 imaging, have limited their utilities. The retina is considered a window to the whole 84 body, implying that the retina could provide clues for ageing.

\section{Added value of this study}

87 We developed a deep learning (DL) model that can detect footprints of aging in

88 fundus images and predict age with high accuracy for the UK population between 40 89 and 69 years old. Further, we have been the first to demonstrate that each one-year 90 increase in retinal age gap (retinal age-chronological age) was significantly associated 91 with a $2 \%$ increase in mortality risk. Evidence of a non-linear association between 92 retinal age gap and mortality risk was observed. Higher retinal age gaps were associated with substantially increased risks of mortality, but only if the retinal age

94 gap exceeded 3.71 years.

95

\section{Implications of all the available evidence}

97 This is the first study to link the retinal age gap and mortality risk, implying that 98 retinal age is a clinically significant biomarker of ageing. Our findings show the potential of retinal images as a screening tool for risk stratification and delivery of

100 tailored interventions. Further, the capability to use fundus imaging in predicting 101 ageing may improve the potential health benefits of eye disease screening, beyond the 102 detection of sight-threatening eye diseases. 


\section{Introduction}

104 Globally, the population aged 60 and over is estimated to reach $2 \cdot 1$ billion in $2050 .^{1}$

105 Ageing populations place tremendous pressure on health-care systems. ${ }^{2}$ The rate of 106 ageing at an individual level is heterogeneous. An accurate quantification of the 107 biological ageing process is significant for risk stratification and the delivery of 108 tailored interventions. ${ }^{3}$

109

110 To date, several tissue-, cell-, molecular-, and imaging-based biomarkers have been 111 developed, such as DNA-methylation status, brain age and three dimensional (3D)

112 facial age. ${ }^{4-7}$ While the invasiveness of cellular and molecular ageing biomarkers, 113 high cost and time-consuming nature of neuroimaging and facial ages, and ethical and 114 privacy concerns of facial imaging, have limited their utilities.

115

116 The retina is considered a window to the whole body. ${ }^{8-12}$ In addition, the retina is 117 amenable to rapid, non-invasive, and cost-effective assessments. The advent of deep 118 learning (DL) has greatly improved the accuracy of image classification and 119 processing. Recent studies have demonstrated successful applications of DL models 120 in the prediction of age using clinical images. ${ }^{5,6,13}$ Taken together, this raises the 121 potential that biological age can be predicted by applying DL to retinal images. For 122 optimal utility, viable biomarkers of ageing must also relate to the risk of age-related 123 morbidity and mortality.

124

125 We therefore developed a DL model that can predict age from fundus images, known 126 as retinal age. Using a large population-based sample of middle-aged and elderly 127 adults, we investigated the association between retinal age gap, defined as the 128 difference between retinal age and chronological age, and all-cause mortality.

\section{Methods}

\section{Study population}


132 The UK Biobank is a large-scale, population-based cohort of more than 500,000 UK

133 residents aged 40-69 years. Participants were recruited between 2006 and 2010, with

134 all participants completing comprehensive health-care questionnaires, detailed

135 physical measurements, and biological sample collections. Health-related events were

136 ascertained via data linkage to hospital admission records and mortality registry.

137 Ophthalmic examinations were introduced in 2009. The overall study protocol and

138 protocols for each test have been described in extensive details elsewhere. ${ }^{14}$

140 The National Information Governance Board for Health and Social Care and the NHS

141 North West Multicenter Research Ethics Committee approved the UK Biobank study

$142(11 / \mathrm{NW} / 0382)$ in accordance with the principles of the Declaration of Helsinki, with

143 all participants providing informed consent. The present analysis operates under UK

144 Biobank application 62525.

145

\section{Fundus photography}

147 Ophthalmic measurements including LogMAR visual acuity, autorefraction and 148 keratometry (Tomey RC5000, Tomey GmbH, Nuremberg, Germany), intraocular 149 pressure (IOP, Ocular Response Analyzer, Reichert, New York, USA), and paired 150 retinal fundus and optical coherence tomography imaging (OCT, Topcon 3D OCT 1511000 Mk2, Topcon Corp, Tokyo, Japan) were collected. A 45-degree non-mydriatic 152 and non-stereo fundus image centered to include both the optic disc and macula was 153 taken for each eye. A total of 131,238 images from 66,500 participants were obtained 154 from the UK Biobank study, among which 80,170 images from 46,970 participants 155 passed the image quality check.

\section{Deep learning model for age prediction}

158 To build the DL model for age prediction, participants from the UK Biobank study 159 alive on $28^{\text {th }}$ February $2018\left(\mathrm{~N}_{\text {subj }}=46,970\right)$ were randomly split into three datasets 160 training $\left(\mathrm{N}_{\text {subj }}=27,424,60 \%\right.$ of participants $)$, validation $\left(\mathrm{N}_{\text {subj }}=9,142,20 \%\right)$, and test $161\left(\mathrm{~N}_{\text {subj }}=9,142,20 \%\right)$. For the training and validation datasets, fundus images from both 
162 eyes (if available) were used to maximise the volume of data available $\left(\mathrm{N}_{\text {img }}=46,834\right.$

163 and 15,612 respectively). For the test dataset, fundus images from right eyes were

164 selected for primary analyses $\left(\mathrm{N}_{\mathrm{subj}}=8,212\right)$, while fundus images from left eyes were 165 selected for sensitivity analyses.

166

167 The development and validation of the DL model for age prediction are outlined in

168 Figure 1. Briefly, all fundus images were preprocessed by subtracting average color, ${ }^{15}$

169 resized to a resolution of $299 * 299$ pixels, and pixel values rescaled to $0 \sim 1$. After 170 preprocessing, images were fed into a DL model using a Xception architecture.

171 During training, data augmentation was performed using random horizontal or 172 vertical flips and the algorithm optimised using stochastic gradient descent. To 173 prevent overfitting, we implemented a dropout of 0.5 , and carried out early stopping 174 when validation performance did not improve for 10 epochs. The selection of DL 175 models was based on performance in the validation set. The performance of the DL 176 model, including mean absolute error (MAE) and correlation between predicted 177 retinal age and chronological age, was calculated. We then retrieved attention maps 178 from the DL models using guided Grad-CAM, ${ }^{16}$ which highlights pixels in the input 179 image based on their contributions to the final evaluation.

\section{Retinal age gap definition}

182 The difference between retinal age predicted by the DL model and chronological age 183 was defined as the retinal age gap. A positive retinal age gap indicated an 'older' 184 appearing retina, while a negative retinal age gap indicated a 'younger' appearing 185 retina.

\section{Mortality ascertainment}

188 Mortality status and date of death were ascertained via data linkage to the National 189 Health Service central mortality registry. Participants who had died from all causes 190 during the follow-up period $\left(\mathrm{N}_{\text {subj }}=1,117\right)$ were included in the death dataset. Duration 191 of follow-up for each participant (person-year) was calculated as the length of time 
192 between baseline age and date of death, loss to follow-up, or complete follow-up ( $28^{\text {th }}$

193 February 2018), whichever came first.

194

195 Covariates

196 Factors previously known to be associated with mortality ${ }^{17}$ were included as potential 197 confounders in the present analyses. These variables included baseline age, sex, 198 ethnicity (recorded as white and non-white), Townsend deprivation indices (an 199 area-based proxy measure for socioeconomic status), education attainment (recorded 200 as college or university degree, and others), smoking status (recorded as 201 current/previous and never), physical activity level (recorded as above 202 moderate/vigorous/walking recommendation and not), general health status (recorded 203 as excellent/good and fair/poor), and comorbidities (obesity, diabetes mellitus, 204 hypertension, history of heart diseases, and history of stroke).

206 Body mass index (BMI) was calculated as body weight in kilograms divided by 207 height squared. Obesity was defined as BMI $\geq 30 \mathrm{~kg} / \mathrm{m}^{2}$. Diabetes mellitus was 208 defined as self-reported or doctor-diagnosed diabetes mellitus, the use of 209 anti-hyperglycaemic medications or insulin, or a glycosylated haemoglobin level 210 of $\geq 6 \cdot 5 \%$. Hypertension was defined as self-reported, or doctor-diagnosed 211 hypertension, the use of antihypertensive drugs, an average systolic blood pressure of 212 at least $130 \mathrm{mmHg}$ or an average diastolic blood pressure of at least $80 \mathrm{mmHg}$. 213 Self-reported history of angina and heart attack was used to classify history of heart 214 diseases.

\section{Statistical analyses}

217 Descriptive statistics, including means and standard deviations (SDs), numbers and 218 percentages, were used to report baseline characteristics of study participants. The 219 retinal age gap was calculated for participants in the test and death datasets, and 220 further used to explore the association between retinal age gap and mortality risk. Cox 221 proportional hazards regression models considering retinal age gap as a continuous 
222 linear term were fitted to estimate the effect of a one-year increase in retinal age gap

223 on mortality risk. We then investigated associations between retinal age gaps at

224 different quantiles with mortality. In addition, a restricted cubic spline analyses of

225 possible non-linear associations between retinal age gap and mortality status was

226 performed, with 5 knots placed at equal percentiles of the retinal age gap, and retinal

227 age gap of zero years used as the reference value. We adjusted Cox models for the

228 following covariates - baseline age, sex, ethnicity, and Townsend deprivation indices

229 (model I); additional educational level, obesity, smoking status, physical activity level,

230 diabetes mellitus, hypertension, general health status, history of heart diseases, and

231 history of stroke (model II).

232

233 The proportional hazards assumption for each variable included in the Cox 234 proportional hazards regression models were graphically assessed. All variables were 235 found to meet the assumption. A two-sided $\mathrm{p}$ value of $<0.05$ indicated statistical 236 significance. Analyses were performed using R (version 3.3.0, R Foundation for 237 Statistical Computing, www.R-project.org, Vienna, Austria) and Stata (version 13, 238 StataCorp, Texas, USA).

239

240 Role of the funding source

241 The funders had no role in study design, data collection, data analyses, data 242 interpretation, preparation of the manuscript, and decision to publish. The 243 corresponding author had full access to all data and final responsibility for the

244 decision to submit for publication.

246 Results

\section{Study sample}

248 The study population characteristics are described in Table 1. The DL model was 249 trained and validated on subsets of participants with mean ages of $55 \cdot 6 \pm 8 \cdot 21$ and $250 \quad 55.7 \pm 8.19$ years; and with $55.9 \%$ and $55.2 \%$ female, respectively. For the test and 
death datasets, participants had mean ages of $55.5 \pm 8.22$ and $61 \cdot 0 \pm 6.67$ years; and were $55.1 \%$ and $42 \cdot 2 \%$ female, respectively.

\section{Deep learning model performance for age prediction}

255 Figure 2A shows the performance of the DL model on the test dataset. The trained DL 256 model was able to achieve a strong correlation of $0.83(\mathrm{P}<0.001)$ between predicted 257 retinal age and chronological age, with an overall MAE of 3.50 years. Two 258 representative examples of fundus images with corresponding attention maps for age 259 prediction are shown in Figure 3. Regions around retinal vessels are highlighted by 260 the DL model for age prediction.

\section{Retinal age gap}

263 The distribution of the retinal age gap followed a nearly normal distribution (Figure 264 2B). The mean (SD) and median (interquartile range) of the retinal age gap were -0.16 265 (4.54) and $-0.19(-2.99,2.60)$. The proportions of fast agers with retinal age gaps more than 3,5 and 10 years were $22.0 \%, 12.0 \%$ and $1.67 \%$, respectively.

\section{Retinal age gap and mortality}

269 Considering linear effects only and following adjustment for all confounding factors, 270 each one-year increase in retinal age gap was associated with a $2 \%$ increase in 271 mortality risk (hazard ratio $[\mathrm{HR}]=1 \cdot 02,95 \%$ confidence interval $[\mathrm{CI}]: 1 \cdot 00-1 \cdot 04, \mathrm{P}=$ 272 0.021; Table 2). Compared to participants with retinal age gaps in the lowest quantile, 273 mortality risk was comparable for those in the second and the third quantiles (HR = $2741 \cdot 05,95 \%$ CI: $0 \cdot 88-1 \cdot 24, \mathrm{P}=0 \cdot 602 ; \mathrm{HR}=0.89,95 \%$ CI: $0 \cdot 73-1 \cdot 09, \mathrm{P}=0 \cdot 261$,

275 respectively). Mortality risk was significantly increased for participants with retinal 276 age gaps in the fourth quantile ( $\mathrm{HR}=1 \cdot 33,95 \% \mathrm{CI}: 1 \cdot 06-1 \cdot 67, \mathrm{P}=0 \cdot 012$; Table 2$)$.

278 Allowing for non-linearity, Figure 5 illustrates the estimated association between 279 retinal age gap and mortality risk. Evidence of an overall and non-linear association 280 between retinal age gap and mortality risk was observed $\left(\mathrm{P}_{\text {overall }}<0.001\right.$; $\mathrm{P}_{\text {non-linear }}=$ 
It is made available under a CC-BY-NC-ND 4.0 International license .

281 0.001). Higher retinal age gaps were associated with substantially increased risks of mortality, but only if the retinal age gap exceeded $3 \cdot 71$ years.

283

284 Sensitivity analyses

285 In order to verify the robustness of our findings, fundus images from left eyes were

286 chosen for the statistical analyses. Similar results were observed for left eyes (data not

287 shown).

288

\section{Discussion}

290 Using a large population-based sample of middle-aged and elderly adults, we

291 developed a DL model that could predict age from fundus images with high accuracy.

292 Further, we found that the retinal age gap, defined as the difference between predicted

293 retinal age and chronological age, independently predicted the risk of mortality. Our

294 findings have demonstrated that retinal age is a robust biomarker of ageing that can

295 predict all-cause mortality.

296

297 To the best of our knowledge, this is the first study that has proposed retinal age as a 298 biomarker of ageing. Our trained DL model achieved excellent performance with a 299 MAE of 3.5, outperforming most existing biomarkers in the prediction of age. 300 Previous studies have demonstrated MAEs of 3.3-5.2 years for DNA methylation 301 clock, ${ }^{18,19} 5 \cdot 5-5 \cdot 9$ years MAEs for blood profiles, ${ }^{20,21}$ and 6-2-7.8 years MAEs for the 302 transcriptome ageing clock. ${ }^{22,23}$ Neuroimaging and 3D facial imaging have achieved 303 accurate performances in age prediction with MAEs between $4 \cdot 3$ and $7 \cdot 3,{ }^{7,24}$ and $2 \cdot 8$ 304 and 6.4 years, ${ }^{6,25}$ respectively. Despite these reasonable accuracies, the invasiveness 305 of cellular and molecular ageing biomarkers, high cost and time-consuming nature of 306 neuroimaging and 3D facial ages, and ethical and privacy concerns of facial imaging, 307 have limited their utilities. In addition to excellent performance in age prediction, 308 determining retinal age using fundus images is fast, safe, cost-effective and 309 user-friendly, thus offering great potential for use in a large number of people. 
311 Beyond age prediction, our study has extended the application of retinal age to the

312 prediction of survival. Our novel findings have determined that the retinal age gap is

313 an independent predictor of increased mortality risk, further suggesting that retinal

314 age is a clinically significant biomarker of ageing. The relevance of retinal age for

315 general health is intuitive, given that the retina is the only organ that is amenable to in

316 vivo visualisation of the microvasculature and neural tissue. The retina offers a unique,

317 accessible 'window' to evaluate underlying pathological processes of systemic

318 vascular and neurological diseases that are associated with increased risks of mortality.

319 This hypothesis is supported by previous studies which have suggested that retinal

320 imaging contains information about cardiovascular risk factors, ${ }^{26}$ chronic kidney

321 diseases ${ }^{27}$ and systemic biomarkers. ${ }^{28}$ In addition, this hypothesis is also consistent

322 with previously reported qualitative and quantitative studies that have found that

323 ocular imaging measures (e.g. retinal-vessel calibre) and retinal diseases (e.g.

324 glaucoma) are significantly associated with mortality. ${ }^{29,30}$ This body of work supports

325 the hypothesis that the retina plays an important role in the ageing process and is

326 sensitive to the cumulative damages of ageing which increase the mortality risk.

328 Our findings have several important clinical implications. Firstly, the fast, 329 non-invasive, and cost-effective nature of fundus imaging enables it to be an 330 accessible screening tool to identify individuals at an increased risk of mortality. This 331 risk stratification will assist tailored health-care decision-making, as well as targeting 332 and monitoring of interventions. Given the rising burden of non-communicable 333 diseases and population ageing globally, the early identification and delivery of 334 personalised health-care may have tremendous public health benefits. Further, the 335 recent development of smartphone-based retinal cameras, together with the 336 integration of DL algorithms, may in the future provide point-of-care assessments of 337 ageing and improve accessibility to tailored risk assessments. Secondly, the capability 338 to use fundus images in predicting ageing may improve potential health benefits of 339 eye disease screening, beyond the diagnosis of sight-threatening eye diseases. This 340 may improve the health economic cost-effectiveness of programs such as diabetic 
341 retinopathy screening, thus increasing the impact and access to eye disease screening

342 programs.

344 The large-scale sample size, long-term follow-up, standardised protocol in capturing

345 fundus images, validity of mortality data, and adjustment for a wide range of

346 confounding factors in the statistical models of this study support the robustness of

347 our findings. Despite these promising results, our study has several limitations. Firstly,

348 these current analyses are limited by retinal images that were captured at a particular

349 cross-section in time, with trajectories in retinal ageing potentially being a better

350 indicator of mortality. Secondly, participants involved in the UK Biobank study were

351 volunteers, who might not be representative of the population from which they were

352 drawn. Of note, the potential healthy effect might underestimate effects of retinal age

353 gap on mortality, as individuals with extremely poor health were less likely to

354 participate in this study. Thirdly, the lack of external datasets might limit the

355 generalisability of our DL algorithms and findings. Lastly, we were unable to fully

356 exclude the possibility of residual confounders between retinal age gap and mortality.

\section{Conclusion}

359 In summary, we have developed a DL algorithm that can detect footprints of ageing in

360 fundus images and predict age with high accuracy. Further, we have been the first to

361 demonstrate that the retinal age gap is significantly associated with an increased risk

362 of mortality. Our findings suggest that retinal age is a robust biomarker of ageing.

363 Lastly, our work calls for future research into applications of the retinal age gap, and

364 whether retinal age can be used to better understand processes underpinning ageing.

\section{Contributors}

367 ZZ and SD conceptualised and designed the study with WW, HM, and YX. ZZ and

368 SD did the literature search and wrote the first draft of the manuscript. SD, PG and

369 MW did the deep learning modelling, ZZ, SX and WW did the statistical analysis. 
370 WW, HM and YX had full access to all of the data. All authors commented on the

371 manuscript.

372

373 Declaration of interests

374 We declare no competing interests.

\section{Acknowledgments}

377 This present work was supported by the NHMRC Investigator Grant (APP1175405),

378 Fundamental Research Funds of the State Key Laboratory of Ophthalmology, 379 National Natural Science Foundation of China (82000901), Project of Investigation 380 on Health Status of Employees in Financial Industry in Guangzhou, China 381 (Z012014075), Science and Technology Program of Guangzhou, China 382 (202002020049). Professor Mingguang He receives support from the University of 383 Melbourne through its Research Accelerator Program and the CERA Foundation. The 384 Centre for Eye Research Australia (CERA) receives Operational Infrastructure 385 Support from the Victorian State Government.

\section{References:}

388 1. United Nations, Department of Economic and Social Affairs, Population Division 389 (2017). World Population Ageing 2017 Available at: 390 https://www.un.org/en/development/desa/population/publications/pdf/ageing/WPA20 391 17_Highlights.pdf [Accessed November 28, 2020].

392 2. MacNee W, Rabinovich RA, Choudhury G. Ageing and the border between 393 health and disease. Eur Respir J 2014; 44(5): 1332-52.

394 3. Lopez-Otin C, Blasco MA, Partridge L, Serrano M, Kroemer G. The hallmarks of 395 aging. Cell 2013; 153(6): 1194-217.

396 4. Horvath S, Raj K. DNA methylation-based biomarkers and the epigenetic clock 397 theory of ageing. Nat Rev Genet 2018; 19(6): 371-84.

398 5. Wang J, Knol MJ, Tiulpin A, et al. Gray Matter Age Prediction as a Biomarker 399 for Risk of Dementia. Proc Natl Acad Sci U S A 2019; 116(42): 21213-8.

400 6. Xia X, Chen $\mathrm{X}, \mathrm{Wu} \mathrm{G}$, et al. Three-dimensional facial-image analysis to predict 401 heterogeneity of the human ageing rate and the impact of lifestyle. Nat Metab 2020; 402 2(9): 946-57.

403 7. Cole JH, Ritchie SJ, Bastin ME, et al. Brain age predicts mortality. Mol 404 Psychiatry 2018; 23(5): 1385-92. 
405

406

407

408

409

410

411

412

413

414

415

416

417

418

419

420

421

422

423

424

425

426

427

428

429

430

431

432

433

434

435

436

437

438

439

440

441

442

443

444

445

446

447

8. Flammer J, Konieczka K, Bruno RM, Virdis A, Flammer AJ, Taddei S. The eye and the heart. Eur Heart J 2013; 34(17): 1270-8.

9. Wong CW, Wong TY, Cheng CY, Sabanayagam C. Kidney and eye diseases: common risk factors, etiological mechanisms, and pathways. Kidney Int 2014; 85(6): 1290-302.

10. London A, Benhar I, Schwartz M. The retina as a window to the brain-from eye research to CNS disorders. Nature reviews Neurology 2013; 9(1): 44-53.

11. Pavkov ME, Harding JL, Chou CF, Saaddine JB. Prevalence of Diabetic Retinopathy and Associated Mortality Among Diabetic Adults With and Without Chronic Kidney Disease. Am J Ophthalmol 2019; 198: 200-8.

12. Kawasaki R, Xie J, Cheung N, et al. Retinal microvascular signs and risk of stroke: the Multi-Ethnic Study of Atherosclerosis (MESA). Stroke 2012; 43(12): 3245-51.

13. Cole JH, Franke K. Predicting Age Using Neuroimaging: Innovative Brain Ageing Biomarkers. Trends Neurosci 2017; 40(12): 681-90.

14. Sudlow C, Gallacher J, Allen N, et al. UK biobank: an open access resource for identifying the causes of a wide range of complex diseases of middle and old age. PLoS Med 2015; 12(3): e1001779.

15. Ebner M. Color constancy based on local space average color. Machine Vision and Applications 2008; 20(5): 283-301.

16. Xu K, Ba J, Kiros R, et al. Show, attend and tell: neural image caption generation with visual attention. arXiv. 2016; (published online April 19.) (preprint) https://arxiv.org/abs/1502.03044 [Accessed November 28, 2020].

17. Ganna A, Ingelsson E. 5 year mortality predictors in 498,103 UK Biobank participants: a prospective population-based study. Lancet 2015; 386(9993): 533-40.

18. Weidner CI, Lin Q, Koch CM, et al. Aging of blood can be tracked by DNA methylation changes at just three $\mathrm{CpG}$ sites. Genome Biol 2014; 15(2): R24.

19. Hannum G, Guinney J, Zhao L, et al. Genome-wide methylation profiles reveal quantitative views of human aging rates. Mol Cell 2013; 49(2): 359-67.

20. Putin E, Mamoshina P, Aliper A, et al. Deep biomarkers of human aging: Application of deep neural networks to biomarker development. Aging (Albany NY) 2016; 8(5): 1021-33.

21. Mamoshina P, Kochetov K, Putin E, et al. Population Specific Biomarkers of Human Aging: A Big Data Study Using South Korean, Canadian, and Eastern European Patient Populations. J Gerontol A Biol Sci Med Sci 2018; 73(11): 1482-90. 22. Peters MJ, Joehanes R, Pilling LC, et al. The transcriptional landscape of age in human peripheral blood. Nat Commun 2015; 6: 8570.

23. Fleischer JG, Schulte R, Tsai HH, et al. Predicting age from the transcriptome of human dermal fibroblasts. Genome Biol 2018; 19(1): 221.

24. Liem F, Varoquaux G, Kynast J, et al. Predicting brain-age from multimodal imaging data captures cognitive impairment. Neuroimage 2017; 148: 179-88.

25. Chen W, Qian W, Wu G, et al. Three-dimensional human facial morphologies as robust aging markers. Cell Res 2015; 25(5): 574-87. 
medRxiv preprint doi: https://doi.org/10.1101/2020.12.24.20248817; this version posted December 30, 2020. The copyright holder for this preprint (which was not certified by peer review) is the author/funder, who has granted medRxiv a license to display the preprint in perpetuity.

It is made available under a CC-BY-NC-ND 4.0 International license .

448

449

450

451

452

453

454

455

456

457

458

459

460

461

462

26. Poplin R, Varadarajan AV, Blumer K, et al. Prediction of cardiovascular risk factors from retinal fundus photographs via deep learning. Nat Biomed Eng 2018; 2(3): 158-64.

27. Sabanayagam C, Xu D, Ting DSW, et al. A deep learning algorithm to detect chronic kidney disease from retinal photographs in community-based populations. The Lancet Digital Health 2020; 2(6): e295-e302.

28. Rim TH, Lee G, Kim Y, et al. Prediction of systemic biomarkers from retinal photographs: development and validation of deep-learning algorithms. The Lancet Digital Health 2020; 2(10): e526-e36.

29. Wang JJ, Liew G, Klein R, et al. Retinal vessel diameter and cardiovascular mortality: pooled data analysis from two older populations. Eur Heart J 2007; 28(16): 1984-92.

30. Khanna RC, Murthy GVS, Giridhar P, et al. Glaucoma-associated long-term mortality in a rural cohort from India: the Andhra Pradesh Eye Disease Study. $\mathrm{Br} J$ Ophthalmol 2018; 102(11): 1477-82.
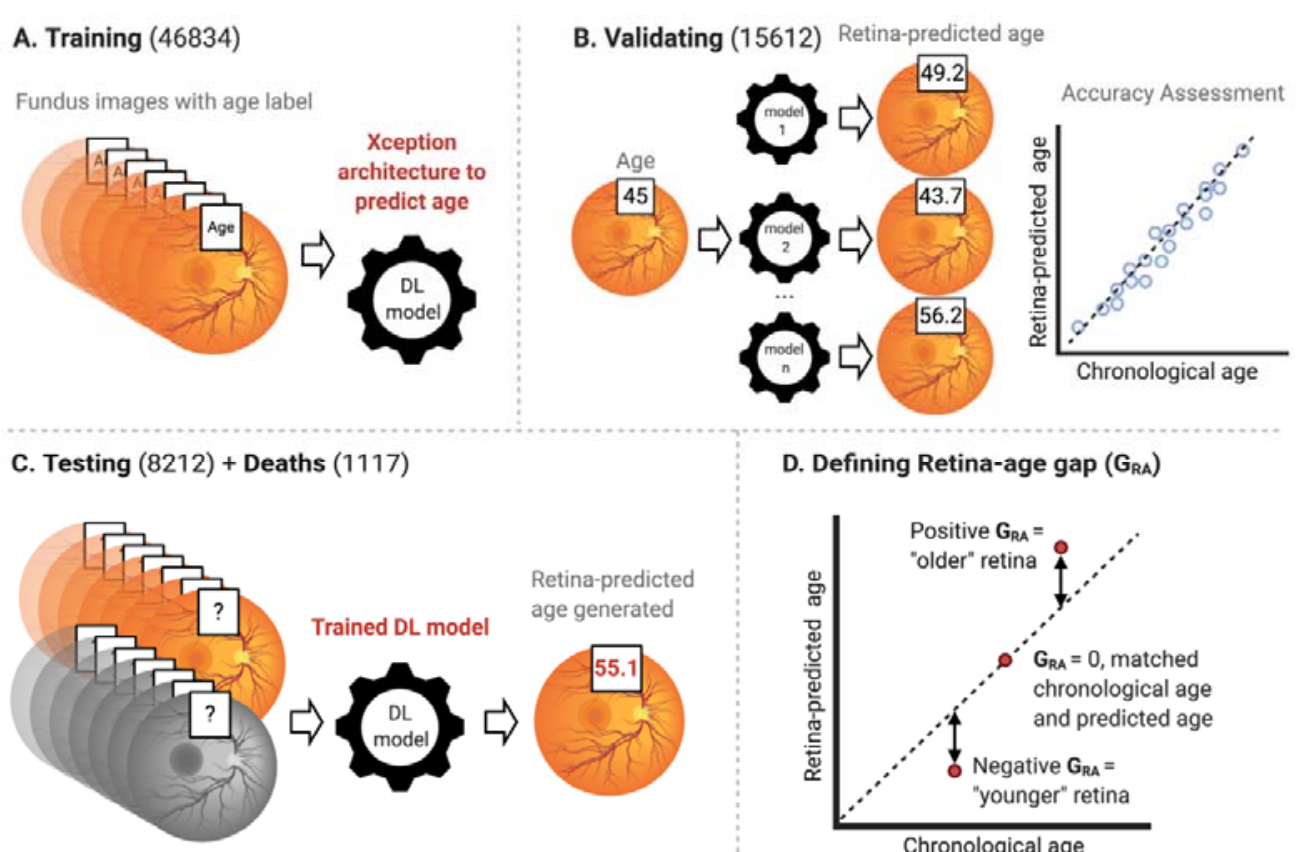

Figure 1. Overview of the study workflow

465 Figure legend: Figures showing the study workflow used to calculate retinal age gaps

466 from fundus images. Fundus images were preprocessed and fed into the DL model. (A)

467 The Xception architecture was used to train fundus images, with chronological age as

468 the outcome variable; (B) The selection of DL models was based on performance in 469 the validation set, where predicted retinal and chronological ages were compared; (C)

470 The selected trained DL model was then applied to make retinal age predictions from 
medRxiv preprint doi: https://doi.org/10.1101/2020.12.24.20248817; this version posted December 30, 2020. The copyright holder for this preprint (which was not certified by peer review) is the author/funder, who has granted medRxiv a license to display the preprint in perpetuity.

It is made available under a CC-BY-NC-ND 4.0 International license .

471 fundus images for participants in the test and death datasets; (D) The difference

472 between predicted retinal age and chronological age was defined as the retinal age gap.

473 A positive retinal age gap indicated an 'older' appearing retina, while a negative

474 retinal age gap indicated a 'younger' appearing retina. This figure was created with

475 BioRender.com.


476

477 Figure 2. Performance of the deep learning model on the test dataset

478 Figure legend: (A) Scatterplot depicting correlation of predicted age (y-axis) with

479 chronological age (x-axis); (B) Histogram showing the nearly normal distribution of 480 the retinal age gap. 
medRxiv preprint doi: https://doi.org/10.1101/2020.12.24.20248817; this version posted December 30, 2020. The copyright holder for this preprint (which was not certified by peer review) is the author/funder, who has granted medRxiv a license to display the preprint in perpetuity.

It is made available under a CC-BY-NC-ND 4.0 International license .

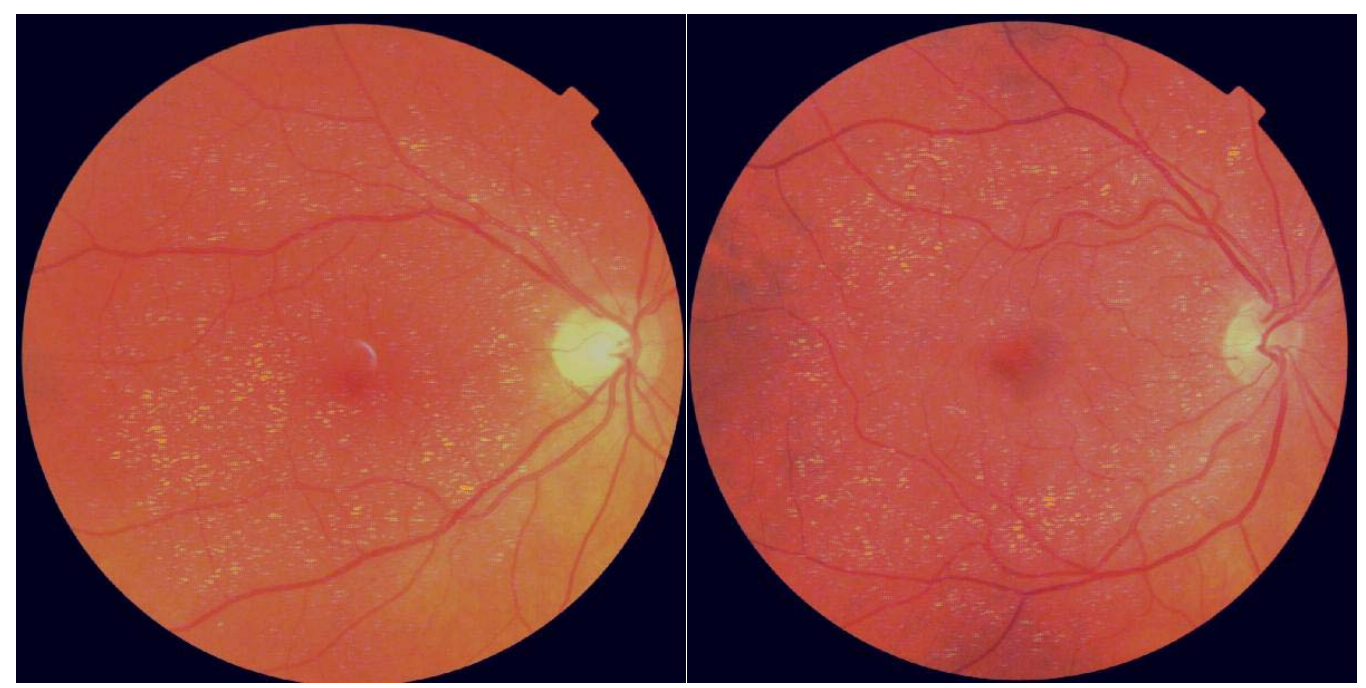

483 Figure 3. Attention maps for age prediction

484 Figure legend: Figures showing representative examples of fundus images with

485 corresponding attention maps for age prediction. Regions highlighted with a brighter 486 colour indicate areas that are used by the DL model for age prediction. Regions 487 around the retinal vessels are highlighted. 


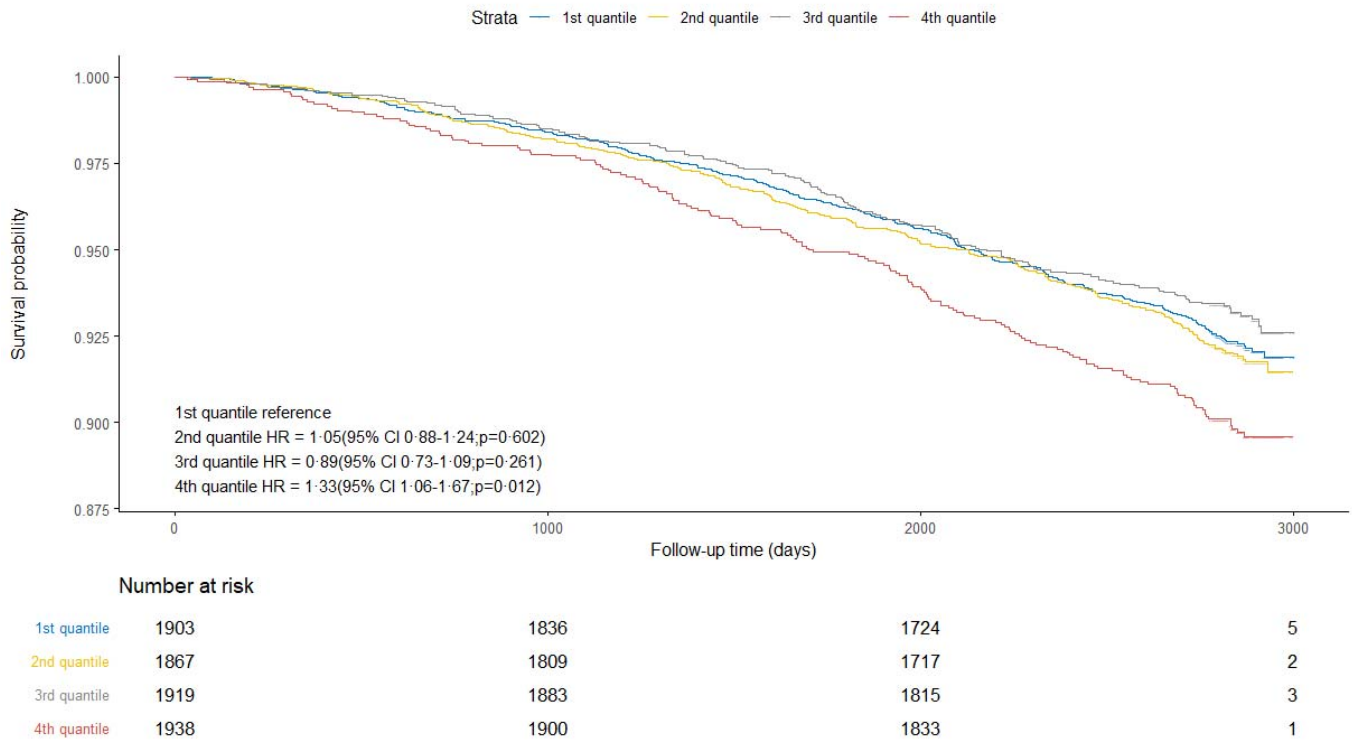

490 Figure 4. Adjusted survival curves for mortality risk by retinal age gap quantiles

491 Figure legend: Mortality risk is shown over time for participants in different retinal 492 age gap quantiles. Lower quantiles corresponded to participants who had 493 chronological ages greater than predicted retinal age, whereas higher quantiles 494 corresponded to those with chronological ages lower than predicted retinal age. Plots 495 were based on Cox proportional hazards regression models, adjusted for age, sex, 496 ethnicity, Townsend deprivation indices, educational level, obesity, smoking status, 497 physical activity level, diabetes mellitus, hypertension, general health status, history 498 of heart diseases, and history of stroke. Compared to participants with retinal age gaps 499 in the lowest quantile, mortality risk was comparable for those in the second and the 500 third quantiles (hazard ratio $[\mathrm{HR}]=1 \cdot 05,95 \%$ confidence interval $[\mathrm{CI}]: 0 \cdot 88-1 \cdot 24, \mathrm{P}$ $501=0 \cdot 602 ; \mathrm{HR}=0 \cdot 89,95 \% \mathrm{CI}: 0 \cdot 73-1 \cdot 09, \mathrm{P}=0 \cdot 261$, respectively). Mortality risk was 502 significantly increased for participants with retinal age gaps in the fourth quantile (HR $503=1 \cdot 33,95 \%$ CI: $1 \cdot 06-1 \cdot 67, \mathrm{P}=0 \cdot 012)$. 


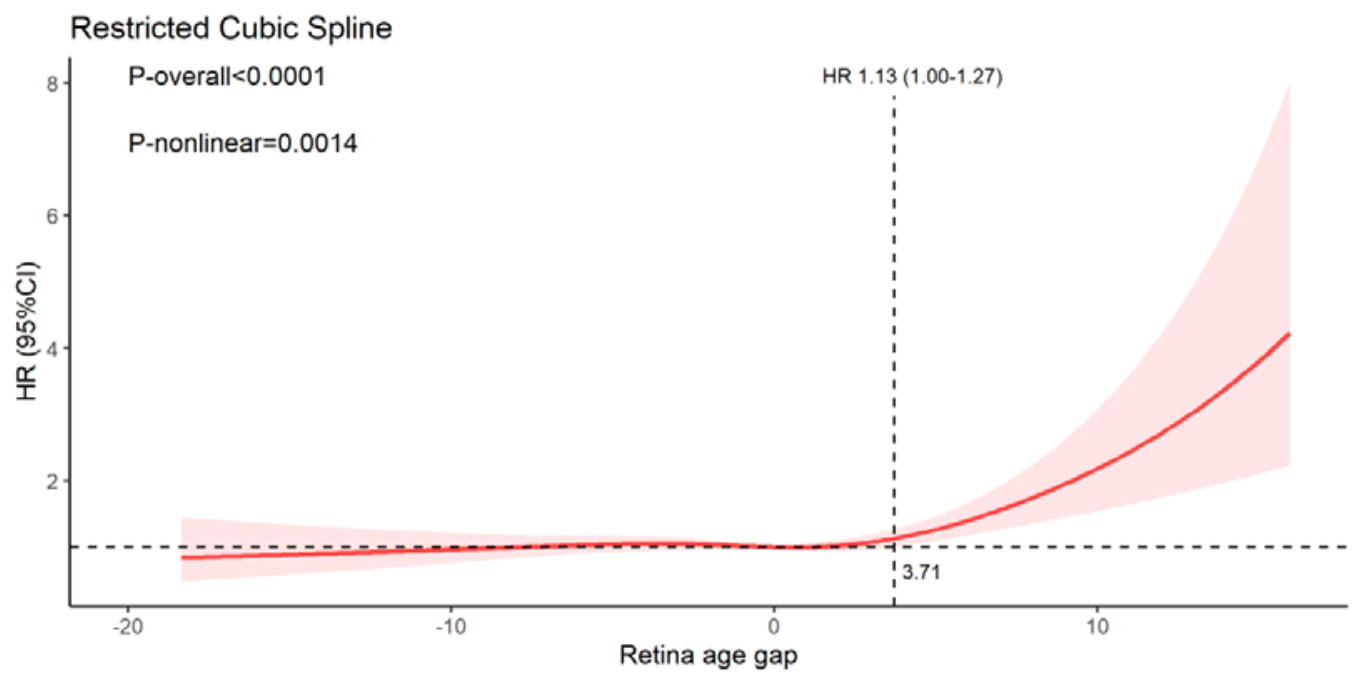

506 Figure 5. Association between retina age gap and mortality risk, allowing for

507 non-linear effects

508 Figure legend: The reference retinal age gap for this plot (with hazard ratio [HR] 509 fixed as 1.0) was 0 years. The model was fitted with a restricted cubic spline for

510 retinal age gap (knots placed at equal percentiles of retina age gap), adjusted for age,

511 sex, ethnicity, Townsend deprivation indices, educational level, obesity, smoking 512 status, physical activity level, diabetes mellitus, hypertension, general health status, 513 history of heart diseases, and history of stroke. Evidence of an overall and non-linear 514 association between retinal age gap and all-cause mortality was observed $\left(\mathrm{P}_{\text {overall }}<\right.$

$\left.5150 \cdot 001 ; \mathrm{P}_{\text {non-linear }}=0 \cdot 001\right)$. Higher retinal age gaps were associated with substantially 516 increased risks of mortality, but only if the retinal age gap exceeded 3.71 years. 
medRxiv preprint doi: https://doi.org/10.1101/2020.12.24.20248817; this version posted December 30, 2020. The copyright holder for this preprint (which was not certified by peer review) is the author/funder, who has granted medRxiv a license to display the preprint in perpetuity.

It is made available under a CC-BY-NC-ND 4.0 International license .

518 Table 1. Characteristics of datasets derived from the UK Biobank study

\begin{tabular}{|c|c|c|c|c|}
\hline & Training $^{a}$ & Validation $^{a}$ & Test & Death \\
\hline $\mathrm{N}_{\text {subj }}$ & 27424 & 9142 & 8212 & 1117 \\
\hline $\mathbf{N}_{\text {img }}$ & 46834 & 15612 & 8212 & 1117 \\
\hline Mean age $(\text { mean } \pm \text { SD, yrs })^{b}$ & $55 \cdot 6 \pm 8 \cdot 21$ & $55 \cdot 7 \pm 8 \cdot 19$ & $55 \cdot 5 \pm 8 \cdot 22$ & $61 \cdot 0 \pm 6 \cdot 67$ \\
\hline Female, $\mathrm{N}(\%)^{\mathrm{b}}$ & $15,341(55 \cdot 9)$ & $5,045(55 \cdot 2)$ & $4,526(55 \cdot 1)$ & $471(42 \cdot 2)$ \\
\hline White ethnicity, $\mathrm{N}(\%)^{\mathrm{b}}$ & $25,400(92 \cdot 6)$ & $8,468(92 \cdot 6)$ & $7,618(92 \cdot 8)$ & $1,035(92 \cdot 7)$ \\
\hline Townsend index $\left(\right.$ mean \pm SD) ${ }^{b}$ & $-1 \cdot 09 \pm 2 \cdot 94$ & $-1 \cdot 10 \pm 2 \cdot 96$ & $-1 \cdot 08 \pm 2 \cdot 95$ & $-0 \cdot 73 \pm 3 \cdot 12$ \\
\hline College or university degree, $\mathrm{N}(\%)^{\mathrm{b}}$ & $9,981(36 \cdot 4)$ & $3,344(36 \cdot 6)$ & $3,035(37 \cdot 0)$ & $299(26 \cdot 8)$ \\
\hline Current/previous smoker, $\mathrm{N}(\%)^{\mathrm{b}}$ & $11,671(42 \cdot 8)$ & $3,905(43 \cdot 0)$ & $3,532(43 \cdot 1)$ & $659(59 \cdot 4)$ \\
\hline Above physical activity recommendation, $\mathrm{N}(\%)^{\mathrm{b}}$ & $18,746(82 \cdot 7)$ & $6,219(82 \cdot 4)$ & $5,682(83 \cdot 4)$ & $681(77 \cdot 4)$ \\
\hline Excellent/good health status, $\mathrm{N}(\%)^{\mathrm{b}}$ & $20,290(74 \cdot 5)$ & $6,738(74 \cdot 0)$ & $6,149(75 \cdot 2)$ & $618(55 \cdot 8)$ \\
\hline Obesity, $\mathrm{N}(\%)^{\mathrm{b}}$ & $6,239(22 \cdot 9)$ & $2,112(23 \cdot 2)$ & $1,840(22 \cdot 5)$ & $312(28 \cdot 1)$ \\
\hline Diabetes mellitus, $\mathrm{N}(\%)^{\mathrm{b}}$ & $1,332(4 \cdot 86)$ & $443(4 \cdot 85)$ & $428(5 \cdot 21)$ & $133(11 \cdot 9)$ \\
\hline Hypertension, $\mathrm{N}(\%)^{\mathrm{b}}$ & $19,632(71 \cdot 6)$ & $6633(72 \cdot 6)$ & $5,956(72 \cdot 5)$ & $928(83 \cdot 1)$ \\
\hline History of heart diseases, $\mathrm{N}(\%)^{\mathrm{b}}$ & $848(3 \cdot 09)$ & $296(3 \cdot 24)$ & $250(3 \cdot 04)$ & $94(8 \cdot 42)$ \\
\hline History of stroke, $\mathrm{N}(\%)^{\mathrm{b}}$ & $309(1 \cdot 13)$ & $83(0 \cdot 91)$ & $103(1 \cdot 25)$ & $35(3 \cdot 13)$ \\
\hline \multicolumn{5}{|c|}{$\mathrm{N}_{\text {subj }}=$ number of subjects; $\mathrm{N}_{\mathrm{img}}=$ number of images; $\mathrm{yrs}=$ years; $\mathrm{SD}=$ standard deviation.} \\
\hline \multicolumn{5}{|l|}{${ }^{a}$ Selection of images of both eyes if available. } \\
\hline${ }^{\mathrm{b}}$ Values are based on $\mathrm{N}_{\text {subj. }}$. & & & & \\
\hline
\end{tabular}


medRxiv preprint doi: https://doi.org/10.1101/2020.12.24.20248817; this version posted December 30, 2020. The copyright holder for this preprint (which was not certified by peer review) is the author/funder, who has granted medRxiv a license to display the preprint in perpetuity.

It is made available under a CC-BY-NC-ND 4.0 International license .

520 Table 2. Association between retinal age gap with mortality using Cox proportional 521 hazards regression models

\begin{tabular}{|c|c|c|c|c|c|c|}
\hline \multirow[b]{2}{*}{ Retinal age gap } & \multirow[b]{2}{*}{$\mathbf{N}$} & \multirow[b]{2}{*}{ Mean+SD (yrs) } & \multicolumn{2}{|l|}{ Model I } & \multicolumn{2}{|l|}{ Model II } \\
\hline & & & HR $(95 \%$ CI $)$ & P value & HR $(95 \% \mathrm{CI})$ & P value \\
\hline Retinal age gap, per one age (yrs) & 9,329 & $-0 \cdot 31 \pm 4 \cdot 59$ & $1 \cdot 03(1 \cdot 01-1 \cdot 05)$ & $<0 \cdot 001$ & $1 \cdot 02(1 \cdot 00-1 \cdot 04)$ & $0 \cdot 021$ \\
\hline \multicolumn{7}{|l|}{ Retinal age gap } \\
\hline Quantile 1 & 2,333 & $-5 \cdot 98+2 \cdot 69$ & Reference & - & Reference & - \\
\hline Quantile 2 & 2,332 & $-1 \cdot 72 \pm 0 \cdot 82$ & $1 \cdot 11(0 \cdot 96-1 \cdot 29)$ & $0 \cdot 168$ & $1 \cdot 05(0 \cdot 88-1 \cdot 24)$ & $0 \cdot 602$ \\
\hline Quantile 3 & 2,332 & $1 \cdot 01 \pm 0 \cdot 81$ & $0 \cdot 96(0 \cdot 80-1 \cdot 15)$ & $0 \cdot 666$ & $0 \cdot 89(0 \cdot 73-1 \cdot 09)$ & $0 \cdot 261$ \\
\hline Quantile 4 & 2,332 & $5 \cdot 43 \pm 2 \cdot 59$ & $1 \cdot 46(1 \cdot 20-1 \cdot 78)$ & $<0 \cdot 001$ & $1 \cdot 33(1 \cdot 06-1 \cdot 67)$ & $0 \cdot 012$ \\
\hline \multicolumn{7}{|c|}{$\mathrm{HR}=$ hazard ratio $; \mathrm{CI}=$ confidence interval.} \\
\hline \multicolumn{7}{|c|}{ Model I adjusted for age, sex, ethnicity, and Townsend deprivation indices. } \\
\hline \multicolumn{7}{|c|}{ Model II adjusted for covariates in Model I + educational level, obesity, smoking status, physical activity level, diabetes mellitus, } \\
\hline
\end{tabular}

